\section{Macular thickness measurements with frequency domain- OCT for quantification of axonal loss in chronic papilledema from pseudotumor cerebri syndrome}

MLR Monteiro and CL Afonso
Division of Ophthalmology, University of São Paulo Medical School, São Paulo, Brazil

Correspondence:

MLR Monteiro, Division of Ophthalmology, University of São Paulo Medical School, Avenue Angélica 1757 conjuncto 61, 01227 200, São Paulo, Brazil. Tel: +55 1136617582 ;

Fax: +551136617582

E-mail: mlrmonteiro@usp.br

Received: 18 August 2013 Accepted in revised form: 25 November 2013

Published online:

10 January 2014

Presented in part at the 38th Annual NeuroOphthalmology Society Meeting in San Antônio, TX USA, 14 February 2012

\begin{abstract}
Purpose To evaluate the ability of frequency domain-optical coherence tomography (FD-OCT)-measured macular thickness parameters to differentiate between eyes with resolved chronic papilledema and healthy eyes and to evaluate the correlation between FD-OCT measures and visual field (VF) loss on standard automated perimetry (SAP). Methods Fifty-two eyes from 29 patients suffering from pseudotumor cerebri syndrome (PTC) and 62 eyes from 31 normal controls underwent FD-OCT scanning and ophthalmic evaluation including VF with SAP. All patients had previously been submitted to treatment of PTC and had clinically resolved papilledema and stable VF for at least 6 months before the study. Macular and peripapillary retinal nerve fiber layer (RNFL) thickness measurements were determined for both groups. Comparisons were made using Generalized Estimated Equations. Correlations between FD-OCT and VF measurements were verified.

Results In eyes with resolved papilledema, the macular thickness parameters corresponding to the inner and outer superior, temporal, inferior and nasal segments, average macular thickness and most RNFL thickness measurements were significantly reduced when compared with controls. The discrimination ability was similar for macular thickness measurements and RNFL thickness measurements. Both sets of OCT measurements correlated well with VF sensitivity loss.
\end{abstract}

Conclusions Eyes with resolved chronic papilledema show a significant reduction in macular thickness, which is well correlated with the severity of VF loss. Macular thickness measurements can potentially be used to estimate and monitor the amount of ganglion cell loss in eyes with papilledema from patients with PTC.

Eye (2014) 28, 390-398; doi:10.1038/eye.2013.301; published online 10 January 2014

Keywords: optical coherence tomography; papilledema; pseudotumor cerebri syndrome; macular thickness measurements

\section{Introduction}

The clinical assessment of anterior visual pathway diseases involves both functional evaluations, such as visual acuity (VA) and visual field (VF) assessments, and structural evaluations of the neural elements of the eye, traditionally optic nerve head $(\mathrm{ONH})$ appearance and, more recently, retinal nerve fiber layer (RNFL) and macular thickness measurements. ${ }^{1,2}$ Studies evaluating the relationship between functional and structural measurements of the neural structures of the eye are of great importance for diagnosis and monitoring the course of the disease. VF loss, which is usually assessed using standard automated perimetry (SAP), is the most commonly used parameter to estimate the severity of visual loss. Structural measurements, on the other hand, can be obtained with a number of technologies, the most common of which is optical 
coherence tomography (OCT), which acquires crosssectional images of retinal structures from which estimates of the neural integrity of the fundus can be made. Over the last years, several studies have shown that OCT is an extremely important tool to detect and quantify structural axonal damage in a number of optic nerve diseases including glaucoma and compressive, inflammatory/ demyelinating, ischemic and hereditary optic neuropathies. ${ }^{2-5}$ Axonal loss is usually quantified with OCT based on peripapillary RNFL thickness measurements; however, measurements of macular thickness can also be used to estimate the amount of retinal ganglion cell loss.

Papilledema is an important cause of progressive and permanent visual loss and RNFL loss, mostly in patients with pseudotumor cerebri syndrome (PTC) ${ }^{6-8}$ a disease that may be defined as raised intracranial pressure without localizing neurological findings, ventriculomegaly, or intracranial tumor. The diagnosis of PTC is currently applied to patients with idiopathic intracranial hypertension (IIH) with no identifiable cause, or to patients with cerebral venous outflow system obstruction or impairment. ${ }^{9}, 10 \mathrm{VF}$ loss on SAP, the main morbidity of PTC, occurs in up to $92 \%$ of eyes. ${ }^{8}$ Although visual loss is initially reversible, when RNFL and RGC atrophy develop, permanent VF loss occurs in a large percentage of eyes.,11-13 Therefore, monitoring visual function is extremely important during the treatment of PTC. Previous studies have indicated that OCTmeasured peripapillary RNFL thickness can be used to estimate the degree of papilledema and, consequently, to monitor treatment efficacy. ${ }^{14-18}$ The presence of optic disc edema, however, artificially increases OCTmeasured RNFL thickness measurements and prevents accurate estimation of peripapillary axonal loss.

In recent years, OCT hardware and software improvements have made it possible to use macular thickness measurements to quantify neuronal loss. As the RGC layer accounts for a significant percentage of the thickness in the macular area, estimates of macular thickness can be used to investigate possible RGC layer loss, as demonstrated in eyes with glaucoma, ${ }^{19-21}$ and compressive $\mathrm{e}^{2,22-24}$ or demyelinating optic

neuropathies. ${ }^{4,25,26}$ However, no study has yet evaluated the ability of macular thickness measurements to estimate axonal loss following papilledema.

The purpose of this study was therefore to evaluate the ability of OCT-measured macular thickness to estimate neural loss in eyes with resolved chronic papilledema from PTC and abnormal VF. We also evaluated the relationship between macular thickness measurements and the severity of VF damage in eyes with papilledema and compared the ability of macular thickness parameters and peripapillary RNFL thickness measurements to differentiate eyes with papilledema from controls.

\section{Patients and methods}

\section{Study design and sampling}

This was an observational, prospective cross-sectional study. Participants were recruited from the Neuroophthalmology service of the University of São Paulo Medical School. Approval from the Institutional Review Board Ethics Committee was obtained for the study. The study followed the principles of the Declaration of Helsinki and informed consent was obtained from all participants.

A total of 52 eyes with clinically resolved papilledema from 29 patients (26 women) diagnosed with PTC and 62 eyes from 31 normal controls (26 women) were evaluated. PTC was diagnosed based on its definition as a syndrome of increased intracranial pressure without ventriculomegaly or a mass lesion, and with normal cerebrospinal fluid (CSF) composition. This included patients with IIH and intracranial hypertension secondary to elevated intracranial venous pressure. ${ }^{10}$ In our sample, 24 patients had IIH and five had PTC secondary to cerebral venous thrombosis. IIH was defined based on previously published criteria, ${ }^{27}$ with high CSF opening pressure (ICP $>25 \mathrm{~cm} \mathrm{H}_{2} \mathrm{O}$ ) measured by lumbar puncture at the time of diagnosis; normal magnetic resonance (MR) imaging and MR venography; normal CSF composition; and normal neurological examination except for papilledema and possible sixth cranial nerve palsy. In patients with cranial sinus thrombosis, the diagnosis was based on neuroimaging studies including MR venography and/or cerebral angiography.

The other inclusion criteria for PTC patients were as follows: (i) clinically resolved papilledema, (grade 0 according to the Frisen Scale ${ }^{28}$ ) with stable VF defect for at least 6 months after clinical and/or surgical treatment, (ii) CSF pressure measurement $<25 \mathrm{~cm} \mathrm{H}_{2} \mathrm{O}$ after papilledema resolution, and (iii) abnormal VF defined as the presence of at least two contiguous test points, not including those directly above and below the blind spot, with both a total deviation (TD plot) and a pattern deviation (PD plot) of one point with $P<0.5 \%$ and another point with $P<2 \%$. Patients with intracranial diseases other than cranial venous sinus thrombosis were excluded. The control group consisted of normal healthy volunteers recruited from among the hospital staff.

\section{Optical coherence tomography}

Subjects underwent frequency domain-OCT (FD-OCT) scanning using commercially available equipment (3D OCT-1000, Topcon Corp., Tokyo, Japan) on the same day as the ophthalmic evaluation. The authors reviewed the images with respect to their subjective 
and objective qualities. The scanning protocol used in this study involved the acquisition of a set of three highdefinition OCT images of the $\mathrm{ONH}$ and macula in a raster pattern covering a 6-mm area with a scan density of $512 \times 128$ pixels in $\sim 3.5 \mathrm{~s}$ (27000 A scans/s) (Figure 1 ). Criteria for acceptable 3D OCT-1000 fundus images included the following: (A) no large eye movements, defined as an abrupt shift completely disconnecting a large retinal vessel; (B) consistent signal intensity level across the scan, and (C) no black bands (caused by blinking) throughout the examination.

Peripapillary RNFL and macular thickness parameters were automatically calculated by the equipment's software. The macular thickness measurements were measured on the Early Treatment Diabetic Retinopathy Study map. The parameters registered in this study were superior outer macular thickness, inferior outer macular thickness, temporal outer macular thickness, nasal outer macular thickness, superior inner macular thickness, inferior inner macular thickness, temporal inner macular thickness, nasal inner macular thickness, and average macular thickness. Average macular thickness corresponded to the weighted average of the sectoral macular thickness measurements excluding the fovea. RNFL thickness measurements were taken using a circular $(\varnothing=3.4 \mathrm{~mm})$ map drawn around the $\mathrm{ONH}$, when taking measurements corresponding to average thickness $\left(360^{\circ}\right)$, and the following $\mathrm{ONH}$ sectors: temporal $\left(310^{\circ}-41^{\circ}\right)$, superotemporal $\left(41^{\circ}-80^{\circ}\right)$, superonasal $\left(80^{\circ}-120^{\circ}\right)$, nasal $\left(121^{\circ}-230^{\circ}\right)$, inferonasal $\left(231^{\circ}-270^{\circ}\right)$, and inferotemporal $\left(271^{\circ}-310^{\circ}\right)$ (Figures $1 \mathrm{a}$ and $\mathrm{b}$ ).

\section{Visual function testing}

Patients and controls underwent a complete ophthalmologic examination, including bestcorrected monocular VA assessment and SAP conducted with a Humphrey Field Analyzer (CarlZeiss Meditec, Dublin, CA, USA) using the Swedish Interactive Threshold Algorithm (SITA standard 24-2 program) and a Goldmann size III stimulus on a 31.5-apostilb background.

The severity of VF defects was evaluated using the mean deviation (MD) provided by the equipment based on data from the TD plot. Deviation from normal at each test location was measured in decibels. Unlogged $1 /$ Lambert (1/L) values were calculated for each test location by dividing the decibel readings by 10 and then unlogging the quotient. Values were averaged in order to calculate the MD in $1 / \mathrm{L}$ units. We also calculated VF sensitivity loss from the 12 central points of the VF, defined as the central mean defect (CMD), from an area
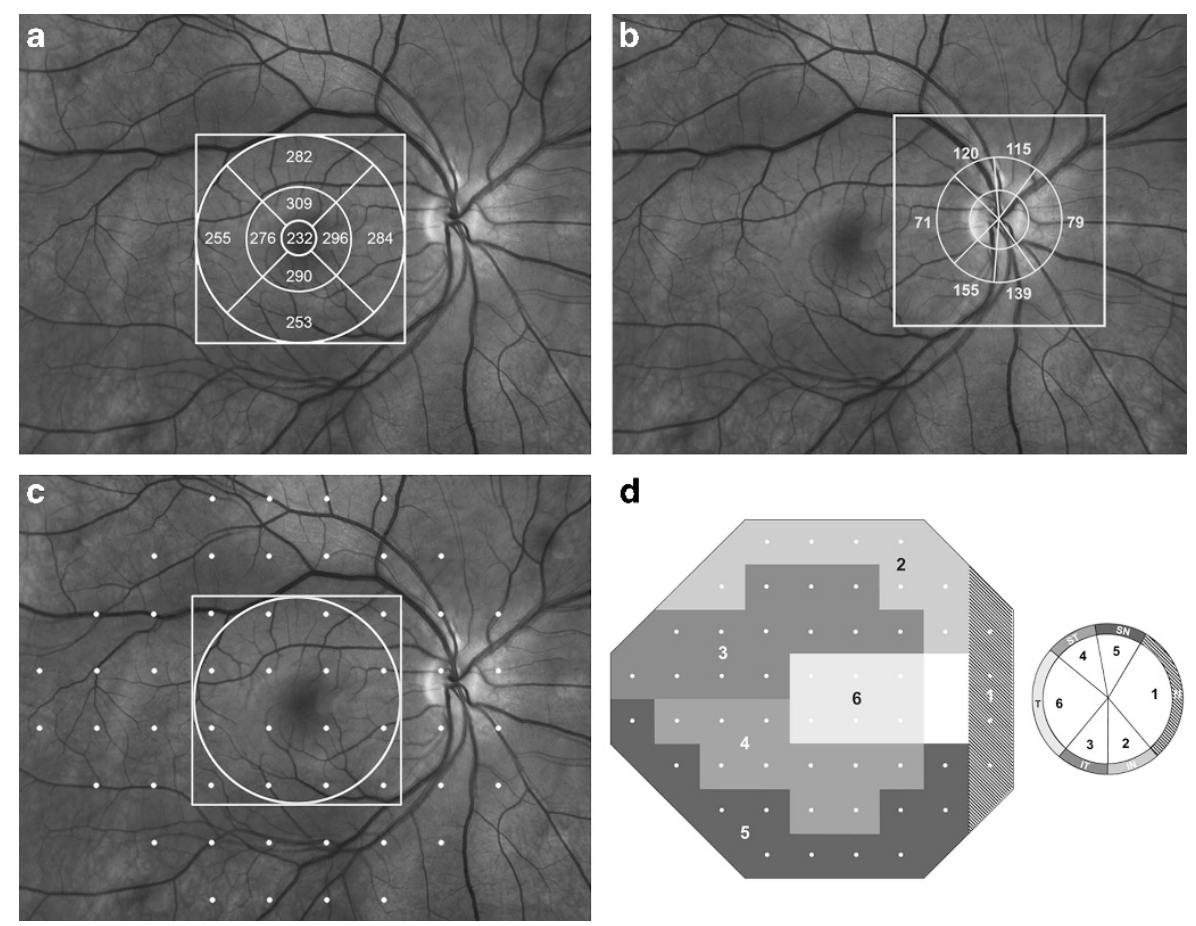

Figure 1 Above: demarcation of areas in the macula (a, outer square line) and optic nerve (b, outer square line) scanned by Fourierdomain-optical coherence tomography with schematic representation of a macular thickness map (a, circle) and RNFL thickness (b, peripapillary circle) of a normal individual. Below: demarcation of points read on 24-2 standard automated perimetry (c). The 12 points contained in the central circle correspond to the area evaluated in the circular macular map. (d): Division of the 24 visual field test points and the optic nerve head into sectors, derived from a published optic disc visual field map. ${ }^{29}$ 
roughly equivalent to the area covered by 3D OCT-1000 raster scanning in the macular area and evaluated by the EDTS circular protocol (Figure 1c). VF sensitivity loss was also determined for six VF sectors according to a previously published optic disc VF map. ${ }^{29}$ These six sectors correspond to the inferonasal, inferotemporal, temporal, superotemporal, superonasal, and nasal optic disc sector (Figure 1d).

For all patients and controls, ophthalmological inclusion criteria for the study were best-corrected VA of $20 / 200$ or better in at least one eye for patients and 20/20 for controls, spherical refraction within $\pm 5 \mathrm{D}$ and cylinder refraction within $\pm 4 \mathrm{D}$, intraocular pressure $<22 \mathrm{~mm} \mathrm{Hg}$, and reliable VF. The ophthalmologic exclusion criteria for patients and controls were history of clinically apparent optic neuropathies other than papilledema (in PTC subjects), history of intraocular pressure elevation, clinical signs of glaucomatous optic neuropathy, and optic disc anomaly.

\section{Data analysis and statistics}

Descriptive statistics included mean \pm SD for normally distributed variables and the median, first and third quartiles, for non-normally distributed variables. Analysis of histograms and the Shapiro-Wilk test were used to evaluate the normality assumption.

OCT measurements of different groups of eyes were compared by using Generalized Estimating Equation (GEE) models to compensate for inter-eye dependencies. In this study, most patients and controls had both eyes included. As eyes of the same individual were expected to have some degree of intercorrelation with respect to VF and OCT parameters, GEE models were used to adjust for within-patient inter-eye correlations. GEE models are generalized linear models that allow for the specification of within-group correlations when evaluating the ability of one or several independent variables to predict a dependent variable. Receiver operating characteristic (ROC) curves were used to describe the ability of OCT parameters to discriminate eyes with chronic papilledema from controls. The method of DeLong et $a l^{30}$ was used to compare the areas under the ROC curves (AUCs).

Pearson's correlation coefficients were used to assess possible associations between OCT and VF sensitivity loss expressed in the $1 / \mathrm{L}$ scale. The level of statistical significance was defined as $P<0.05$.

\section{Results}

A total of 114 eyes were evaluated, of which 52 were from 29 patients with PTC and 62 were from 31 healthy controls. The mean age \pm SD was $40.4 \pm 9.7$ years (range: 26-56) in PTC patients and $38.2 \pm 12.2$ years (range: $21-63)$ in normal subjects $(P=0.44$; Student's $t$-test). On SAP, 14 eyes of patients with PTC had glaucomatous hemifield test results within normal limits, four were borderline, and 34 were outside normal limits. The median (first quartile and third quartile) SAP MD and the central MD were $-4.5 \mathrm{~dB}(-10.2,-2.3)$ and $-2.9 \mathrm{~dB}(-7.4 \mathrm{~dB},-1.8 \mathrm{~dB})$. The fundoscopic examination revealed either normal appearance (20 eyes) or signs of resolved papilledema with varying degrees of optic disc pallor (32 eyes). Six eyes of patients with PTC were excluded: three because VA was worse than 20/200, two because VF revealed no abnormalities, and one due to the presence of myelinated nerve fibers around the disc.

Figure 2 depicts examples of FD-OCT macular and RNFL thickness measurements in patients with PTC. Table 1 shows macular thickness measurements in eyes with PTC and control eyes. All macular thickness parameters were significantly lower in eyes with PTC than in normal eyes $(P<0.05, \mathrm{GEE})$, except for the values corresponding to the inferior inner macular segment. ROC curve areas and sensitivities at fixed specificities are shown in Table 1. The macular thickness of the outer nasal (AUC $=0.72)$, outer temporal (AUC $=0.71$ ), and outer superior $(\mathrm{AUC}=0.71)$ segments had the largest AUCs.

Table 2 shows average and sectoral RNFL measurements for patients with PTC and controls. The two groups differed significantly with regard to each study parameter $(P<0.05, \mathrm{GEE})$ except for the inferonasal segment. ROC curve areas and sensitivities at fixed specificities are also shown in Table 2. The RNFL thickness parameters corresponding to the superotemporal (AUC $=0.80)$, average $(A U C=0.75)$, and inferotemporal or nasal (AUC $=0.70)$ thickness measurements had the largest AUCs. No significant difference was observed between the best-performing macular and RNFL thickness parameters.

Table 3 shows the correlation between average or sectoral macular/RNFL thickness and VF sensitivity loss (in 1/L units) assessed globally (MD) in 12 central test points (CMD) and in six VF areas. Significant correlations between OCT and VF findings were found for most macular and peripapillary RNFL thickness measurements. With macular thickness measurements, the three most significant correlations $(P<0.001)$ were between VF sector 5 and the average, inner temporal, or outer nasal macular segments $(r=0.47$ each). With RNFL thickness, the three most significant correlations were between average thickness and VF sector 5 or 6 $(r=0.55$ each $)$ and between average thickness and VF sector $6(r=0.54)$. No significant difference was observed between the best-performing macular and RNFL thickness parameter and VF loss. 
a
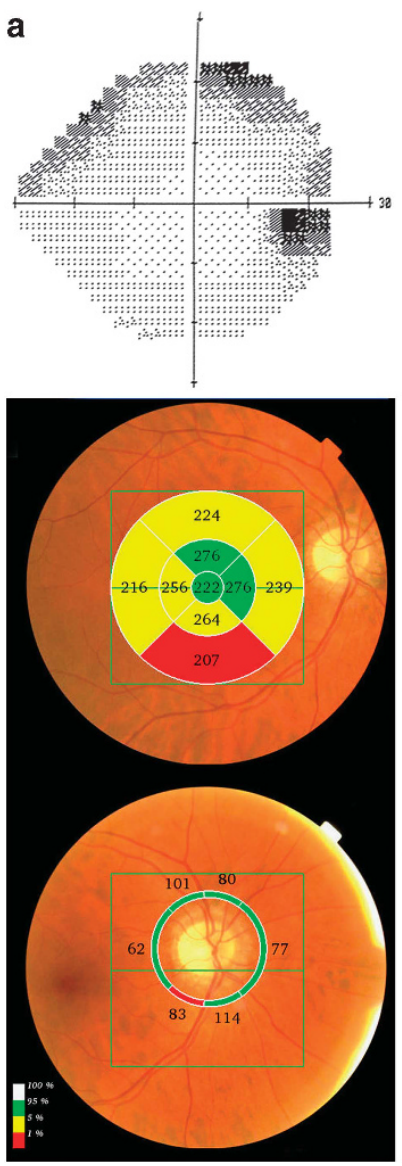

b
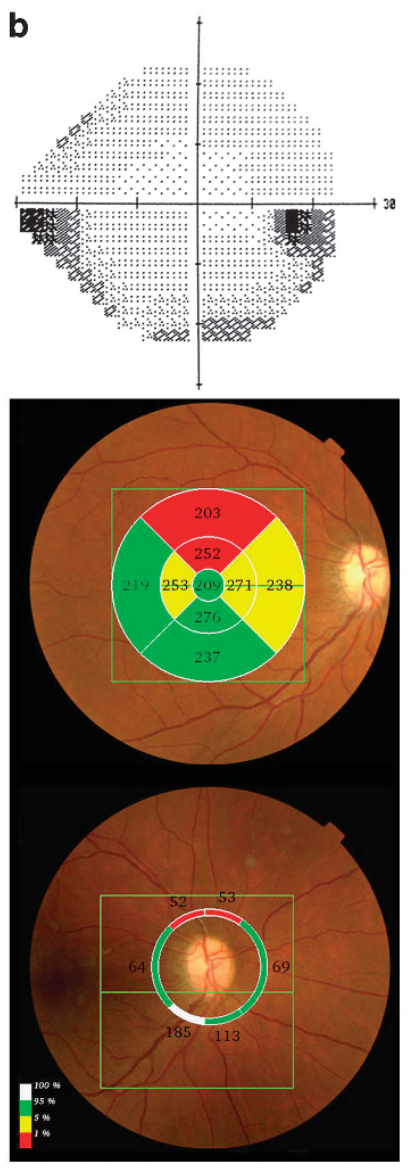

c
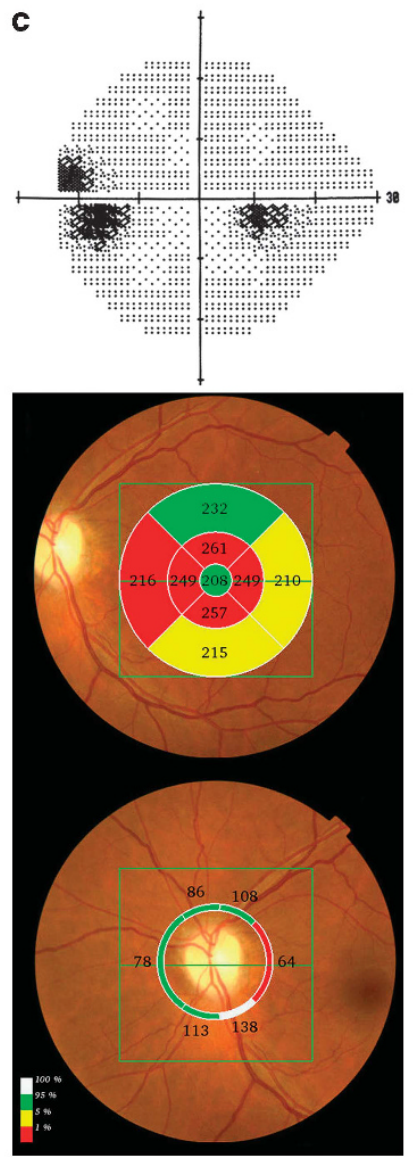

d
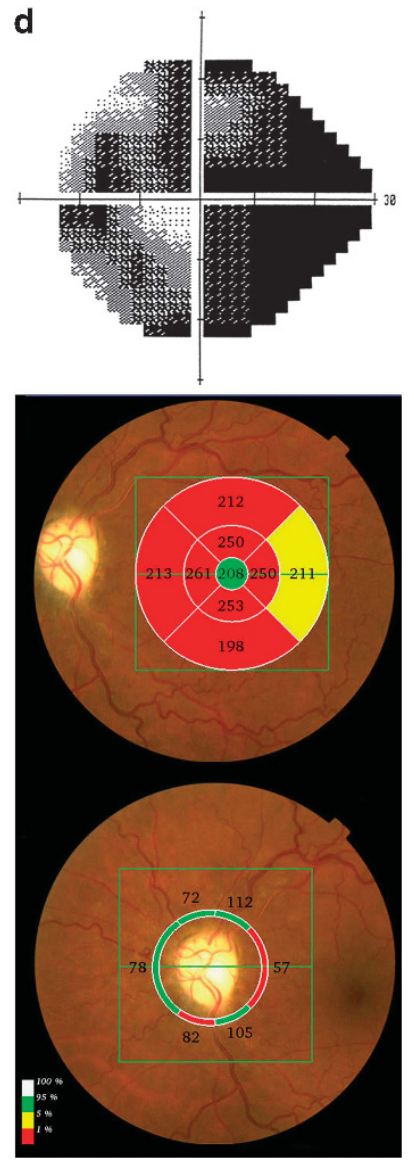

Figure 2 Examples of visual field (upper row) and FD-OCT macular (second row) and RNFL (third row) thickness measurements generated by the Topcon 3D OCT-1000 equipment in four eyes (a-d) with resolved papilledema from pseudotumor cerebri different degrees of visual field loss. Measurements in different sectors are indicated with numbers and represented in colors that correspond to the normal distribution. Sectors in green indicate values within the normal range; in yellow less than the 5th, in red less than the 1st and in white more than the 95th percentile compared with an age-matched reference population.

\section{Discussion}

In this study we evaluated a large number of eyes from patients with resolved papilledema from PTC. Our results document the ability of OCT to quantify axonal loss in this condition, as there was a significant reduction in most macular and RNFL thickness measurements when compared with normals. These findings are in agreement with clinical observations of optic disc pallor and peripapillary RNFL loss that may occur as a result of chronic papilledema. ${ }^{6}$ It is widely known that the main morbidity of PTC syndrome is visual impairment, which can be severe and permanent, in 10-72\% of eyes, depending on the series. ${ }^{711-13}$ It is therefore of great importance to monitor the visual function of PTC patients during treatment. Whereas visual loss in papilledema may be reversible at early stages, permanent visual loss may occur.
Estimating the amount of retinal axonal loss is of great interest during treatment of PTC syndrome, particularly when treatment achieves only partial control of raised intracranial pressure and incomplete resolution of papilledema. Medical treatment of PTC is based on dieting for obese patients, the use acetazolamide for reducing intracranial pressure, and anticoagulants for cranial sinus thrombosis. Surgical treatment, either with optic nerve sheath fenestration or shunting procedures, may be necessary when visual loss occurs despite medical treatment. The treatment decision is usually straightforward; however, when the treatment fails to achieve complete control of PTC and complete resolution of papilledema, the decision whether to continue medical treatment or perform surgery can be difficult to make and is usually based on VF analyses. However, VF assessment in itself does not indicate the possibility of recovering vision, which is dependent on the amount 
Table 1 Mean values ( \pm SD) of frequency domain optical coherence tomography macular full thickness parameters (in $\mu \mathrm{m}$ ) with areas under the receiver operating characteristic curves (AUC) and sensitivities at fixed specificities

\begin{tabular}{lcccc}
\hline Parameter & $\begin{array}{c}\text { Pseudotumor } \\
\text { cerebri } \\
(\mathrm{n}=52)\end{array}$ & $\begin{array}{c}\text { Controls } \\
(\mathrm{n}=62)\end{array}$ & Pa & AUC (SE) \\
\hline Average thickness sectors & $258.6 \pm 19.0$ & $271.5 \pm 13.7$ & 0.001 & $0.69(0.05)$ \\
Superior inner & $285.6 \pm 22.4$ & $297.1 \pm 17.5$ & 0.02 & $0.64(0.05)$ \\
Temporal inner & $269.8 \pm 21.4$ & $281.5 \pm 16.4$ & 0.01 & $0.66(0.05)$ \\
Inferior inner & $282.8 \pm 21.7$ & $290.0 \pm 18.0$ & 0.12 & $0.59(0.05)$ \\
Nasal inner & $285.4 \pm 25.8$ & $297.6 \pm 18.0$ & 0.02 & $0.62(0.05)$ \\
Superior outer & $244.3 \pm 18.3$ & $256.0 \pm 15.3$ & 0.001 & $0.71(0.05)$ \\
Temporal outer & $229.4 \pm 14.1$ & $241.2 \pm 14.1$ & $<0.001$ & $0.71(0.05)$ \\
Inferior outer & $238.4 \pm 19.8$ & $251.3 \pm 16.7$ & 0.003 & $0.67(0.05)$ \\
Nasal outer & $257.3 \pm 25.8$ & $277.1 \pm 16.4$ & $<0.001$ & $0.72(0.05)$ \\
\hline
\end{tabular}

Significant values are in italic.

${ }^{\text {a }}$ Generalized estimated equation.

Table 2 Mean values $( \pm S D)$ of frequency domain optical coherence tomography retinal nerve fiber layer thickness parameters (in $\mu \mathrm{m}$ ) with areas under the receiver operating characteristic curves (AUC) and sensitivities at fixed specificities

\begin{tabular}{lrccc}
\hline Parameter & $\begin{array}{c}\text { Pseudotumor } \\
\text { cerebri } \\
(\mathrm{n}=52)\end{array}$ & $\begin{array}{c}\text { Normal } \\
(\mathrm{n}=62)\end{array}$ & Pa & AUC (SE) \\
\hline Average thickness & $96.2 \pm 16.7$ & $108.1 \pm 7.4$ & $<0.001$ & $0.75(0.05)$ \\
Superotemporal thickness & $115.0 \pm 24.6$ & $139.6 \pm 12.8$ & $<0.001$ & $0.80(0.04)$ \\
Temporal thickness & $74.3 \pm 14.9$ & $80.2 \pm 11.4$ & 0.04 & $0.61(0.05)$ \\
Inferotemporal thickness & $125.6 \pm 27.7$ & $143.5 \pm 17.9$ & 0.001 & $0.70(0.05)$ \\
Inferonasal thickness & $116.1 \pm 32.1$ & $125.9 \pm 16.7$ & 0.11 & $0.61(0.06)$ \\
Nasal thickness & $86.0 \pm 18.4$ & $95.8 \pm 13.8$ & 0.004 & $0.70(0.05)$ \\
Superonasal thickness & $105.4 \pm 31.4$ & $119.4 \pm 16.6$ & 0.02 & $0.67(0.05)$
\end{tabular}

Significant values are in italics.

${ }^{a}$ Generalized estimated equations (GEE).

of existing axonal loss. Furthermore, patients with IIH may have concurrent functional visual loss, making it even more difficult to estimate visual function by perimetry alone. ${ }^{31}$ Ney et al ${ }^{31}$ have shown that 11 (65\%) of 17 patients with IIH had functional visual loss clearly confounding the management of the disease. The authors pointed out the mismatch in such patients between the progression of VF loss and the absence of atrophic disc changes. Patients with such difficulties in performing VF may be submitted to unnecessary or even risky surgical procedures due to the inability to clearly document the efficacy of the treatment.

Therefore, using methods for assessing retinal axonal loss in patients receiving treatment for papilledema due to PTC is highly desirable.

Using high-contrast RNFL photographs and VF of 36 eyes with papilledema from IIH, Hedges III et al found RNFL changes in $67 \%$ of eyes. The authors pointed out that the evaluation of RFNL provides objective information regarding the status of the optic nerve and helps to improve clinical management. However, RNFL cannot be accurately quantified by photographic assessment. The development of technologies with which RNFL loss may be quantified, including OCT, scanning laser polarimetry, and confocal scanning laser ophthalmoscopy (CSLO), has been an important landmark in the diagnosis and management of a number of optic nerve diseases. Nevertheless, although these technologies have been used to diagnose and monitor optic disc edema in patients with raised intracranial hypertension, ${ }^{15,16,18,32-38}$ very few studies have attempted to quantify axonal loss in eyes with papilledema. Using CSLO and automated perimetry, Salgarello $e t a l^{34}$ evaluated eight patients with recently diagnosed papilledema and found a significant correlation between the amount of disc edema and VF loss. Laemmer et $a l^{35}$ investigated 23 patients with IIH using scanning laser polarimetry (GDx VCC) and found, compared with normals, an increase in RNFL thickness in patients with papilledema and a decrease in RNFL in 10 out of 13 patients with regression of papilledema. The authors pointed out that even in patients with effective treatment and regression of papilledema, axonal loss can be detected by scanning laser polarimetry. As for OCT, while several studies have evaluated its ability to quantify optic disc edema, ${ }^{18,37}$ none have used it to estimate axonal loss following papilledema.

The most important limitation of many of the equipments used in the quantification of axonal loss in papilledema is the difficulties introduced by the presence of disc edema itself. As most devices quantify RNFL in terms of thickness, the presence of swollen nerve fibers due to axonal transport blockage in papilledema can confound results (Figure 3). Thus, a reduction in RNFL thickness measurements in a patient under treatment for PTC could be due to either a reduction in raised intracranial pressure (indicating an improvement of the condition) or axonal loss of RGC (indicating a worsening of the condition). ${ }^{38}$

Our study is important in that it documents the ability of OCT-measured macular thickness measurements to estimate axonal loss following papilledema. In our study, OCT macular abnormalities in resolved papilledema from PTC were similar to RNFL thickness measurement reductions. Thus, while macular thickness was reduced in relation to controls in eight out of nine macular parameters, RNFL thickness measurements were abnormal in six of seven RNFL thickness parameters (Tables 2 and 3). In addition, the ROC curve analysis indicates similar discrimination ability for macular thickness and RNFL thickness measurements in eyes of PCS patients (Tables 2 and 3). Although our patients were evaluated in the resolved stage of papilledema, it is reasonable to assume that macular thickness evaluations 
Table 3 Relationship between OCT macular and retinal nerve fiber layer (RNFL) thickness and visual field parameters in different field sectors

\begin{tabular}{|c|c|c|c|c|c|c|c|c|}
\hline \multirow{3}{*}{ OCT thickness measurement } & \multicolumn{8}{|c|}{ Visual field parameter (1/Lambert units) } \\
\hline & \multirow[b]{2}{*}{$M D$} & \multirow[b]{2}{*}{$C M D$} & \multicolumn{6}{|c|}{ Visual field sector } \\
\hline & & & 1 & 2 & 3 & 4 & 5 & 6 \\
\hline \multicolumn{9}{|l|}{ Macula (full thickness) } \\
\hline Average & $\underline{0.43}$ & $\underline{0.43}$ & 0.39 & 0.31 & 0.41 & 0.42 & $\underline{0.47}$ & $\underline{0.47}$ \\
\hline Superior inner & $\overline{0.41}$ & $\overline{0.38}$ & 0.34 & 0.33 & 0.40 & 0.40 & $\overline{0.45}$ & $\underline{0.44}$ \\
\hline Temporal inner & $\underline{0.43}$ & 0.42 & 0.34 & 0.35 & $\underline{0.44}$ & 0.40 & $\underline{0.47}$ & $\underline{0.46}$ \\
\hline Inferior inner & $\overline{0.38}$ & 0.41 & 0.33 & 0.30 & $\overline{0.39}$ & 0.34 & $\overline{0.39}$ & 0.42 \\
\hline Nasal inner & 0.41 & 0.42 & 0.36 & 0.31 & 0.40 & 0.36 & $\underline{0.45}$ & $\underline{0.46}$ \\
\hline Superior outer & 0.38 & 0.33 & 0.32 & 0.26 & 0.36 & $\underline{0.43}$ & $\overline{0.42}$ & 0.37 \\
\hline Temporal outer & 0.37 & 0.37 & 0.32 & 0.28 & 0.33 & $\overline{0.39}$ & 0.41 & 0.37 \\
\hline Inferior outer & 0.34 & 0.38 & 0.33 & 0.20 & 0.32 & 0.33 & 0.39 & 0.37 \\
\hline Nasal outer & 0.41 & 0.43 & $\underline{0.45}$ & 0.25 & 0.37 & 0.40 & $\underline{0.47}$ & $\underline{0.48}$ \\
\hline \multicolumn{9}{|l|}{ Peripapillary RNFL } \\
\hline Average & $\underline{0.49}$ & $\underline{0.52}$ & $\underline{0.49}$ & 0.30 & $\underline{0.45}$ & $\underline{0.45}$ & $\underline{0.55}$ & $\underline{0.55}$ \\
\hline Nasal & 0.26 & 0.27 & 0.36 & 0.09 & 0.20 & 0.29 & 0.31 & 0.32 \\
\hline Superonasal & 0.35 & 0.36 & 0.43 & 0.15 & 0.29 & 0.32 & 0.37 & 0.41 \\
\hline Superotemporal & $\underline{0.47}$ & 0.38 & 0.37 & 0.28 & 0.40 & $\underline{0.54}$ & $\underline{0.54}$ & 0.42 \\
\hline Temporal & 0.37 & $\underline{0.44}$ & 0.35 & 0.19 & 0.32 & 0.30 & $\overline{0.42}$ & $\underline{0.46}$ \\
\hline Inferotemporal & $\underline{0.46}$ & $\underline{0.46}$ & 0.38 & 0.42 & $\underline{0.51}$ & 0.30 & $\underline{0.45}$ & $\underline{0.44}$ \\
\hline Inferonasal & $\overline{0.44}$ & $\overline{0.50}$ & 0.31 & 0.34 & $\overline{0.45}$ & 0.35 & $\overline{0.47}$ & 0.47 \\
\hline
\end{tabular}

Abbreviations: CMD, central mean deviation; MD, mean deviation.

Pearson's correlation coefficients. Italic $=P<0.05$; bold $P<0.01$; bold and underlined $=P \leq 0.001$.
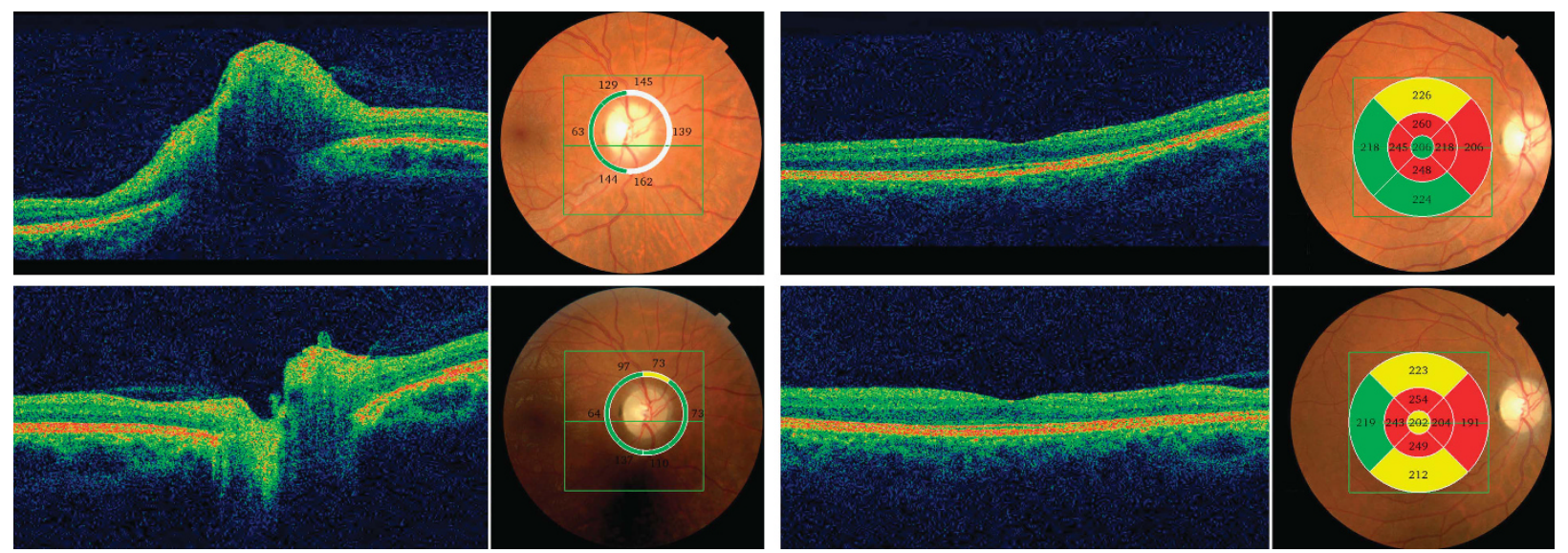

Figure 3 Thickness measurements generated by Topcon 3D OCT-1000 equipment in the same eye in the presence of optic disc edema (upper row) and after resolution of papilledema (lower row). Sectors in green indicate values within normal range; in yellow less than the 5th, in red less than the 1st and in white more than the 95th percentile compared with an age-matched reference population. Note that peripapillary RNFL is either normal or above normal while optic disc edema is present (upper left) but macular thickness measurements demonstrate axonal loss (upper right). After resolution of optic disc edema, both peripapillary RNFL (lower left) and macular thickness (lower right) demonstrate measurements below normal range.

are likewise useful for estimating retinal axonal loss in the active phase of the condition (with disc edema).

In the current study, we also investigated the relationship between FD-OCT and SAP in PTC patients using both global and sectoral measurements. Whereas some previous studies have evaluated the relationship between the degree of papilledema and SAP, no previous investigations have evaluated the correlation between VF 
loss and retinal axonal loss. In our study, a significant structure-function relationship was observed between VF loss and both macular and RNFL thickness measurements. Whereas many VF parameters were significantly correlated, the best correlations were found between VF defect in the inferior and nasal VF region (areas 5) and OCT-measured macular and RNFL thickness measurements.

The main limitation of the rationale of the present study is the presence of confounding factors when macular thickness measurements are used as an indicator of axonal loss in acute papilledema. In fact, in acutely raised intracranial hypertension, macular edema and hemorrhage may occur, potentially interfering with OCTmeasured macular thickness parameters. Furthermore, total macular thickness measurements also include RNFL in the macular area, which could have some swelling in the acute phase. However, the main use of macular thickness measurements would be during treatment of chronic cases where macular thickness measurements would most likely reflect axonal integrity (Figure 3). In such cases it would be very unlikely for RNFL to be swollen in the macular area. Future studies evaluating patients with PTC before and after resolution of papilledema are necessary to clarify this issue.

Our study shows that macular OCT thickness measurements can provide important information regarding retinal axonal loss in patients with papilledema from PTC. Although axonal loss was documented in the chronic phase of papilledema, our findings, to be confirmed in future prospective studies, suggest a possible use of OCT during treatment of PTC syndrome.

\section{Summary}

What was known before

- Evaluating the relationship between functional and structural measurements of the neural structures of the eye is of great importance for diagnosis and monitoring the course of the papilledema in patients with PTC.

- VF usually assessed by SAP is the most commonly used parameter to estimate the severity of visual loss but does not in itself indicate whether visual loss is permanent or reversible.

- Quantitive measurement of retinal axonal loss in eyes with papilledema is therefore highly desirable.

\section{What this study adds}

- OCT abnormalities in eyes with resolved papilledema showed significant reduction in macular thickness, which correlates with VF loss, indicating that macular thickness measurements can potentially be used to estimate ganglion cell loss in eyes with papilledema from pseudotumor cerebri.

\section{Conflict of interest}

The authors declare no conflict of interest.

\section{Acknowledgements}

This study was supported by grants from Fundação de Amparo a Pesquisa do Estado de São Paulo FAPESP (No 2009/50174-0), São Paulo, Brazil and from Conselho Nacional de Desenvolvimento Científico e Tecnológico, CNPq (No 306487/2011-0), Brasília, Brazil.

\section{References}

1 Danesh-Meyer HV, Carroll SC, Foroozan R, Savino PJ, Fan J, Jiang $Y$ et al. Relationship between retinal nerve fiber layer and visual field sensitivity as measured by optical coherence tomography in chiasmal compression. Invest Ophthalmol Vis Sci 2006; 47: 4827-4835.

2 Monteiro ML, Cunha LP, Costa-Cunha LV, Maia Jr OO, Oyamada MK. Relationship between optical coherence tomography, pattern electroretinogram and automated perimetry in eyes with temporal hemianopia from chiasmal compression. Invest Ophthalmol Vis Sci 2009; 50: 3535-3541.

3 Monteiro ML, Leal BC, Moura FC, Vessani RM, Medeiros FA. Comparison of retinal nerve fibre layer measurements using optical coherence tomography versions 1 and 3 in eyes with band atrophy of the optic nerve and normal controls. Eye (Lond) 2007; 21: 16-22.

4 Monteiro ML, Fernandes DB, Apostolos-Pereira SL, Callegaro D. Quantification of retinal neural loss in patients with neuromyelitis optica and multiple sclerosis with or without optic neuritis using Fourier-domain optical coherence tomography. Invest Ophthalmol Vis Sci 2012; 53: 3959-3966.

5 Barboni P, Savini G, Parisi V, Carbonelli M, La Morgia C, Maresca A et al. Retinal nerve fiber layer thickness in dominant optic atrophy measurements by optical coherence tomography and correlation with age. Ophthalmology 2011; 118: 2076-2080.

6 Hedges 3rd TR, Legge RH, Peli E, Yardley CJ. Retinal nerve fiber layer changes and visual field loss in idiopathic intracranial hypertension. Ophthalmology 1995; 102: 1242-1247.

7 Corbett JJ, Savino PJ, Thompson HS, Kansu T, Schatz NJ, Orr LS et al. Visual loss in pseudotumor cerebri. Follow-up of 57 patients from five to 41 years and a profile of 14 patients with permanent severe visual loss. Arch Neurol 1982; 39: 461-474.

8 Wall M, George D. Idiopathic intracranial hypertension. A prospective study of 50 patients. Brain 1991; 114(Pt 1A): 155-180.

9 Binder DK, Horton JC, Lawton MT, McDermott MW. Idiopathic intracranial hypertension. Neurosurgery 2004; 54: 538-551, discussion 51-52.

10 Fraser C, Plant GT. The syndrome of pseudotumour cerebri and idiopathic intracranial hypertension. Curr Opin Neurol 2011; 24: 12-17.

11 Orcutt JC, Page NG, Sanders MD. Factors affecting visual loss in benign intracranial hypertension. Ophthalmology 1984; 91: 1303-1312. 
12 Wall M, George D. Visual loss in pseudotumor cerebri. Incidence and defects related to visual field strategy. Arch Neurol 1987; 44: 170-175.

13 Monteiro ML. Visual loss in pseudotumor cerebri. Arq Bras Oftalmol 1994; 57: 122-125.

14 Savini G, Bellusci C, Carbonelli M, Zanini M, Carelli V, Sadun AA et al. Detection and quantification of retinal nerve fiber layer thickness in optic disc edema using stratus OCT. Arch Ophthalmol 2006; 124: 1111-1117.

15 Scott CJ, Kardon RH, Lee AG, Frisen L, Wall M. Diagnosis and grading of papilledema in patients with raised intracranial pressure using optical coherence tomography vs clinical expert assessment using a clinical staging scale. Arch Ophthalmol 2010; 128: 705-711.

16 Skau M, Milea D, Sander B, Wegener M, Jensen R. OCT for optic disc evaluation in idiopathic intracranial hypertension. Graefes Arch Clin Exp Ophthalmol 2011; 249: 723-730.

17 El-Dairi MA, Holgado S, O’Donnell T, Buckley EG, Asrani S, Freedman SF. Optical coherence tomography as a tool for monitoring pediatric pseudotumor cerebri. J AAPOS 2007; 11: $564-570$.

18 Kaufhold F, Kadas EM, Schmidt C, Kunte H, Hoffmann J, Zimmermann $\mathrm{H}$ et al. Optic nerve head quantification in idiopathic intracranial hypertension by spectral domain OCT. PLoS One 2012; 7: e36965.

19 Guedes V, Schuman JS, Hertzmark E, Wollstein G, Correnti A, Mancini R et al. Optical coherence tomography measurement of macular and nerve fiber layer thickness in normal and glaucomatous human eyes. Ophthalmology 2003; 110: 177-189.

20 Medeiros FA, Zangwill LM, Bowd C, Vessani RM, Susanna Jr R, Weinreb RN. Evaluation of retinal nerve fiber layer, optic nerve head, and macular thickness measurements for glaucoma detection using optical coherence tomography. Am J Ophthalmol 2005; 139: 44-55.

21 Sung KR, Wollstein G, Kim NR, Na JH, Nevins JE, Kim CY et al. Macular assessment using optical coherence tomography for glaucoma diagnosis. Br J Ophthalmol 2012; 96(12): 1452-1455.

22 Costa-Cunha LV, Cunha LP, Malta RF, Monteiro ML. Comparison of Fourier-domain and time-domain optical coherence tomography in the detection of band atrophy of the optic nerve. Am J Ophthalmol 2009; 147: 56-63, e2.

23 Moura FC, Medeiros FA, Monteiro ML. Evaluation of macular thickness measurements for detection of band atrophy of the optic nerve using optical coherence tomography. Ophthalmology 2007; 114: 175-181.

24 Monteiro ML, Costa-Cunha LV, Cunha LP, Malta RF. Correlation between macular and retinal nerve fibre layer Fourier-domain OCT measurements and visual field loss in chiasmal compression. Eye (Lond) 2010; 24: 1382-1390.

25 Saidha S, Syc SB, Ibrahim MA, Eckstein C, Warner CV, Farrell SK et al. Primary retinal pathology in multiple sclerosis as detected by optical coherence tomography. Brain 2011; 134: 518-533.

26 Ratchford JN, Quigg ME, Conger A, Frohman T, Frohman E, Balcer LJ et al. Optical coherence tomography helps differentiate neuromyelitis optica and MS optic neuropathies. Neurology 2009; 73: 302-308.

27 Friedman DI, Jacobson DM. Diagnostic criteria for idiopathic intracranial hypertension. Neurology 2002; 59: 1492-1495.

28 Frisen L. Swelling of the optic nerve head: a staging scheme. J Neurol Neurosurg Psychiatry 1982; 45: 13-18.

29 Garway-Heath DF, Poinoosawmy D, Fitzke FW, Hitchings RA. Mapping the visual field to the optic disc in normal tension glaucoma eyes. Ophthalmology 2000; 107: 1809-1815.

30 DeLong ER, DeLong DM, Clarke-Pearson DL. Comparing the areas under two or more correlated receiver operating characteristic curves: a nonparametric approach. Biometrics 1988; 44: 837-845.

31 Ney JJ, Volpe NJ, Liu GT, Balcer LJ, Moster ML, Galetta SL. Functional visual loss in idiopathic intracranial hypertension. Ophthalmology 2009; 116: 1808-1813, e1.

32 Waisbourd M, Leibovitch I, Goldenberg D, Kesler A. OCT assessment of morphological changes of the optic nerve head and macula in idiopathic intracranial hypertension. Clin Neurol Neurosurg 2011; 113: 839-843.

33 Rebolleda G, Munoz-Negrete FJ. Follow-up of mild papilledema in idiopathic intracranial hypertension with optical coherence tomography. Invest Ophthalmol Vis Sci 2009; 50: 5197-5200.

34 Salgarello T, Falsini B, Tedesco S, Galan ME, Colotto A, Scullica L. Correlation of optic nerve head tomography with visual field sensitivity in papilledema. Invest Ophthalmol Vis Sci 2001; 42: 1487-1494.

35 Laemmer R, Heckmann JG, Mardin CY, Schwab S, Laemmer AB. Detection of nerve fiber atrophy in apparently effectively treated papilledema in idiopathic intracranial hypertension. Graefes Arch Clin Exp Ophthalmol 2010; 248: 1787-1793.

36 Heckmann JG, Faschingbauer F, Lang C, Reulbach U, Dutsch M, Mardin CY et al. Laser scanning tomography measurement of the extent of papilledema in the follow-up examination of patients with idiopathic intracranial hypertension. J Neurosurg 2007; 107: 543-547.

37 Wang JK, Kardon RH, Kupersmith MJ, Garvin MK. Automated quantification of volumetric optic disc swelling in papilledema using spectral-domain optical coherence tomography. Invest Ophthalmol Vis Sci 2012; 53: 4069-4075.

38 Mulholland DA, Craig JJ, Rankin SJ. Use of scanning laser ophthalmoscopy to monitor papilloedema in idiopathic intracranial hypertension. Br J Ophthalmol 1998; 82: 1301-1305. 